\title{
Unexplained infertility. The perilous voyage of the human oocyte
} \author{
Krishnamoorthy ${ }^{1}$, Ella Ekogha Nsa Mve Rose ${ }^{4}$ and Graziella Badulli ${ }^{*}$ \\ ${ }^{1}$ CEFERMAD (Centre de Fertilité de Madagascar) Lot VK 25 bis, Ambohimanoro, Antananarivo 101, Madagascar \\ ${ }^{2}$ Service de Réanimation Chirurgicale-Hôpital Universitaire Ravoahangy, Antananarivo, Madagascar \\ ${ }^{3}$ Department of Obstetrics and Gynecology, Wellkin Hospital, Mauritius \\ ${ }^{4}$ Centre de Fértilité Ibékélia Marie, Libreville, Gabon
}

Rakotobe Andriamaro Andriantsirombaka ${ }^{1}$, Marcienne Aimée ${ }^{1}$, Ramarolahy Rija ${ }^{2}$, Shilpa Sinha ${ }^{3}$, Leonardo Formigli ${ }^{1}$, Ramachandran

\begin{abstract}
We are able to check and investigate almost all causes of infertility in the male and female. However, the voyage of the oocyte cumulus complex (OCC) from the inner side of the ovarian follicle to the tubal lumen is difficult to monitor and investigate. This journey may occur successfully every month in some women, whereas it may occur very rarely or never in other women and in some others a successful voyage may occur randomly. All this in the presence of normal patent tubes and normal ovulations. Clinical studies have shown that as many as 25 to more than $40 \%$ of oocytes are being trapped inside their empty follicles. It is therefore tempting to speculate that at least in some women their unexplained infertility may be due to a defect of oocyte release from the follicle. Also, a defective pick up by the tubes may threaten the oocyte journey. Studies in laboratory animals show that the administration of prostaglandins may increase the hyaluronic acid embedding among the granulosa cells of the OCC with consequent expansion of the OCC, which helps detaching the OCC from the inner follicular wall and also indirectly increases the quality of the oocyte. A pharmacologic protocol with prostaglandin administration is therefore suggested for unexplained infertility cases.
\end{abstract}

Couples complaining of infertility are commonly advised to wait one to two years, depending on their age, before starting the investigations to determine the reason of their infertility, unless there are obvious causes such as amenorrhoea, previous vasectomy, lack of sexual intercourses and so on.

Once the allowed time is over, patients usually perform sperm tests, a test for assessing the patency of the Fallopian tubes and hormonal assays for the assessment of ovulation. These tests are deemed to identify the causes in most cases of infertility. Some patients, where the infertility diagnosis has still not been made, undergo a diagnostic laparoscopy mainly to ascertain whether there are endometriotic nodules or pelvic adhesions which may prevent the oocyte-cumulus complexes (OCC) to enter into the Fallopian tubes. If even laparoscopy gives a negative response, it is presumed that the oocytes or the spermatozoa may have some genetic/chromosomal abnormalities not allowing a good interaction, or the endometrium may not offer a good environment to the embryos for implantation.

However, an entire aspect of possible infertility namely the perilous voyage of the oocyte from the ovary to the tube is usually taken for granted. When ovulation is monitored by ultrasound, the collapse of the follicle is the sign that ovulation has taken place and it is usually taken for granted that the oocyte has also left the ovary and has entered inside the fallopian tube.

However, the rupture of follicles does not necessarily mean that the oocytes have been released and even less that the oocytes have been captured by the oviductal fimbria. From the diagnostic point of view, detachment of the OCC from the follicular inner wall may be sometimes diagnosed by the ultrasonographic identification of the OCC at the lower pole of the follicle in the hours before ovulation [1].
OCC, once detached from the inner follicular wall, tends to sink to the bottom of the follicle: therefore, if the OCC is freed and free to swim in the follicular fluid, it should be located by ultrasound at the lower pole of the follicle.

A team performing nonsurgical uterine lavage for the recovery of embryos was able to recover oocytes/embryos only in $40 \%$ of the ruptured follicles in natural cycles; in $40 \%$ of ruptured follicles in clomiphene citrate stimulated cycles and in $31 \%$ of ruptured follicles in human menopausal gonadotropin stimulated cycles [2]. Apart from the empty follicle syndrome $[3,4]$, which is pretty rare, less than $1 \%$, there is a chance that in collapsed follicles, OCCs may remain trapped inside the follicles: OCCs may not detach from the inner follicular wall. In fact, contrarily to what is generally thought, emptying of the follicle and release of the OCC are different events, in the sense that the follicle may empty, but the OCC may not be extruded from the follicle. When oocyte pick up (OPU) was performed by laparoscopy during the early years of In Vitro Fertilization (IVF), not infrequently the procedure was performed too late when ovulation had already occurred, or part of the follicles had already ruptured. Laparoscopists used to flush each

*Correspondence to: Graziella Badulli, CEFERMAD (Centre de Fertilité de Madagascar) Lot VK 25 bis, Ambohimanoro, Antananarivo 101, Madagascar, E-mail: graziellaparda@yahoo.it

Key words: failed release of oocyte, failed capture of oocyte, human, ovarian follicle, ovulation, empty follicle, unexplained infertility, prostaglandins, hyaluronan, hyaluronic acid, expansion of oocyte cumulus complex, detachment from inner follicular wall, misoprostol, Cytotec, ovarian pregnancy, mice

Received: March 23, 2020; Accepted: March 30, 2020; Published: April 02, 2020 
collapsed follicle, by entering into the follicle through the follicular stigma. Oocytes surrounded by their cumulus were found on average in 20 to $25 \%$ of the ruptured follicles $[5,6]$.

In a recent study, as many as $43 \%$ of retained oocytes were aspirated vaginally under ultrasound guidance from ruptured follicles in single follicle non stimulated or minimally stimulated IVF procedures [7].

Ovarian pregnancies are probably due to retained oocytes inside the follicles which are being fertilized by the spermatozoa reaching the oocytes through the ovarian stigma [8].

It has been shown in animal studies that an impaired mucification of the cumulus oophorus results in an impaired extrusion of the oocytes from the follicles and in impaired pick up by the oviductal fimbria [9]. Mucification is due to the action of hyaluronan embedding amidst the granulosa cell layers around the oocytes. Thus the cumulus cells undergo considerable expansion, detachment from the follicular inner wall and become also more "sticky" and more easily captured by the ciliated cells of the tubal fimbria. This mucification phenomenon starts one to two days before ovulation.

Prostaglandins dose-dependently stimulate cumulus expansion and detachment in mice [10]. Among all the classes of prostaglandins, the drug Misoprostol (Prostaglandin E1 analogue) is a cheap, readily available and commonly used drug in Obstetrics-induction of labour and abortion [11], Gynecology-dilation of the cervix for hysteroscopy and other procedures [12] and Reproductive Medicine-prevention of the ovarian hyperstimulation syndrome [13]. There is thus a reasonable possibility that the administration of Misoprostol (Cytotec) intravaginally, one to two days before ovulation, at the dose of 800 mcg in a single administration, which is the dose used for preventing hyperstimulation in stimulated cycles, may be useful for increasing the hyaluronic acid within the follicle, expansion of the cumulus cells and help detachment of the OCCs. On the other hand, the danger that prostaglandins may be harmful to a possible pregnancy, due to their luteolytic action, should be negligible, since the luteolytic action acts only on mature corpora lutea: one to two days before ovulation, the corpora lutea are not yet formed, or follicle luteinisation has just started [14]. In addition, it may be argued by some that prostaglandins may inhibit ovum capture by the distal oviduct, or have a negative effect on the oocyte or on the first steps of fertilization, but this is contradicted by others [15].

In women follicles empty their fluid into the peritoneal cavity and by gravity follicular fluid reaches the Douglas pouch, while the OCCs are either directly captured by the oviducts if the stigma are covered by the fimbria, or they fall down into the Douglas pouch, where they are captured by the oviductal fimbria soaking into the follicular fluid filling the cul de sac [16]. Whatever the precise mechanism of pick up by the tubes, it is evident that many OCCs may be lost in the abdominal cavity, since the transit from the ovary to the tube is aleatory and perilous. In fact, contrarily to what happens in many animals species where the tubes are directly connected to the ovary by a membranous duct "bursa ovarica", in the human there are no such ducts. In addition, the abdominal organs filling the pelvis are crammed and it is easy to speculate that, even with a normal pelvis, a bowel loop or omentum may interfere with the physiological process of ovum pick-up by the tube.

Therefore, a substantial number of oocytes/embryos are lost either because they remain trapped in the follicles, or because they get lost in the peritoneal cavity. It is almost impossible to monitor the voyage of the oocyte from the follicle into the tubal lumen and this journey is more or less fortuitous and perilous. In some women a successful voyage occurs almost at every ovulation, in other women the journey occurs less frequently and may give rise to unexplained infertility. This is not a definite cause of infertility, but in some women who get surprisingly pregnant after many years of unexplained infertility there may be a defect in the voyage of their oocytes. In the human, nature entrusts monthly reproduction to the male by way of millions of spermatozoa, whereas the female must put all the hopes in only one very precious, single cell, the oocyte.

In the last decades, since the advent of IVF and even before, stimulating the ovaries for the production of many oocytes has characterized most infertility therapies, with the hope that at least one among the several oocytes produced may succeed. To our knowledge, few if any studies in the human have been focused into facilitating the voyage of the oocyte from the follicle to the tube. Achieving this goal may reduce the overwhelming use of ovarian stimulation in the treatment of human infertility.

It may be suggested to administer prostaglandins, as depicted above, to those patients waiting one to two years. Usually these patients are not happy to wait passively for such a long time and wish to "do something". Thus, administering the prostaglandins every other month, for example, may also help these patients psychologically. The alternate month administration may also help to evaluate the efficacy of this therapy. Also, in case of unexplained infertility, the depicted therapy may be used two or three times before starting Artificial Reproduction Techniques.

Misoprostol, $800 \mathrm{mcg}$ intravaginally in a single administration, may also be administered concomitantly with the ovulation triggering Human Chorionic Gonadotropin administration during stimulated cycles coupled with natural intercourse or intrauterine insemination. One cannot rule out the possibility to use prostaglandins even in IVF cycles, with the aim to induce the development of well expanded cumuluses since it is common knowledge that a well expanded cumulus normally houses a good quality oocyte.

And in the end, since in some cases, OCC may be picked up by the tubes from the Douglas pouch, patients should be asked not to assume a prone position during the days and nights around ovulation, since oocytes may either not reach the Douglas pouch, or may be displaced from the pouch and be lost among the intestinal loops.

\section{Conflict of interest}

The authors report no conflict of interest.

\section{Funding}

The authors report no funding whatsoever.

\section{Contribution of authors}

Conception of the study: RAA, GB, RR, LF. Literature search: SS, MA, RK. Preparation of manuscript: RR

\section{Acknowledgments}

The authors thank the staff of CEFERMAD (Centre de Fertilite de Madagascar), namely: Nathalie Razaiarisoa.

\section{References}

1. Poehl M, Hohlagschwandtner M, Doerner V, Dillinger B, Feichtinger W (2000) Cumulus assessment by three-dimensional ultrasound for in vitro fertilization. Ultrasound Obstet Gynecol 16: 251-253. [Crossref] 
2. Formigli L, Roccio C, Belotti G, Stangalini A, Coglitore MT, et al. (1990) Non-surgical flushing of the uterus for pre-embryo recovery: possible clinical applications. Hum Reprod 5: 329-335.

3. Tanaka R, Murase M, Aizawa Y, Seguio M, Ishidera Y, et al. (2013) A case of empty follicle syndrome who conceived after aspiration of an endometrial cyst. Reprod Med Biol 12: 111-115. [Crossref]

4. Stevenson TL, Lashen H (2008) Empty follicle syndrome: the reality of a controversia syndrome, a systematic review. Fertil Steril 90: 691-698. [Crossref]

5. Craft I, Shelton K, Yovich J and Smith D (1980) Ovum retention in the human. Fertil Steril 34: 537-541. [Crossref]

6. Stanger JD, Yovich J L (1984) Failure of human oocyte release at ovulation. Fertil Steril 41: 827-832. [Crossref]

7. Teramoto S, Asada H, Shozu M (2019) Prematurely ruptured dominant follicles often retain competent oocytes in infertile women. Sci Rep 9: 15041

8. Bontis J, Grimbizis G, Tarlatzis B, Miliaras D, Bili H (1997) Intrafollicular ovarian pregnancy after ovulation induction / intrauterine insemination: pathophysiological aspects and diagnostic problems. Hum Reprod 12: 376-378. [Crossref]

9. Fülöp C, Szantos S, Mukhopadhyay D, Bardos T, Kamath R, et al. (2003) Impaired cumulus mucification and female sterility in tumor necrosis factor-induced protein-6 deficient mice. Development 130: 2253-2261
10. Takahashi T, Igarashi H, Amita M, Hara S, Kurachi H (2010) Roles of prostaglandins during oocyte maturation. Lessons from knockout mice. J Mamm Ova Res 27: 1120

11. Alfirevic Z, Keeney E, Dowswell T, Welton NJ, Dias S, et al. (2015) Labour induction with prostaglandins: a systematic review and network meta-analysis. BMJ 350: 217

12. Fouda UM, Gal Allah SH, Elshaer HS (2016) Optimal timing of misoprostol administration in nulliparous women undergoing office hysteroscopy: a randomized double-blind placebo-controlled study. Fertil Steril 106: 196-201. [Crossref]

13. Formigli L, Badulli G, Ramarolahy R, Aimee M, Krishnamoorthy R, et al. (2019) Misoprostol (Cytotec) for prevention of ovarian hyperstimulation syndrome in cases of in vitro fertilization. Clin Obstet Gynecol Reprod Med 5: 1-2.

14. Archibald LT, Tran T, Massey R, Klapsfein E (1992) Conception rate in dairy cows after timed-insemination and simultaneous treatment with gonadotropin-releasing hormone and/or prostaglandin F2 $\alpha$. Theriogenology 37: 723-726. [Crossref]

15. Suginami H, Yano K, Watanabe K, Matsuura S (1986) A factor inhibiting ovum capture by the oviductal fimbriae present in endometriosis peritoneal fluid. Fertil Steril 44 1140-1146. [Crossref]

16. Croxatto HB (2002) Physiology of gamete and embryo transport through the Fallopian tube. Reprod Biomed Online 4: 160-169. [Crossref]

Copyright: (C2020 Andriantsirombaka RA. This is an open-access article distributed under the terms of the Creative Commons Attribution License, which permits unrestricted use, distribution, and reproduction in any medium, provided the original author and source are credited. 